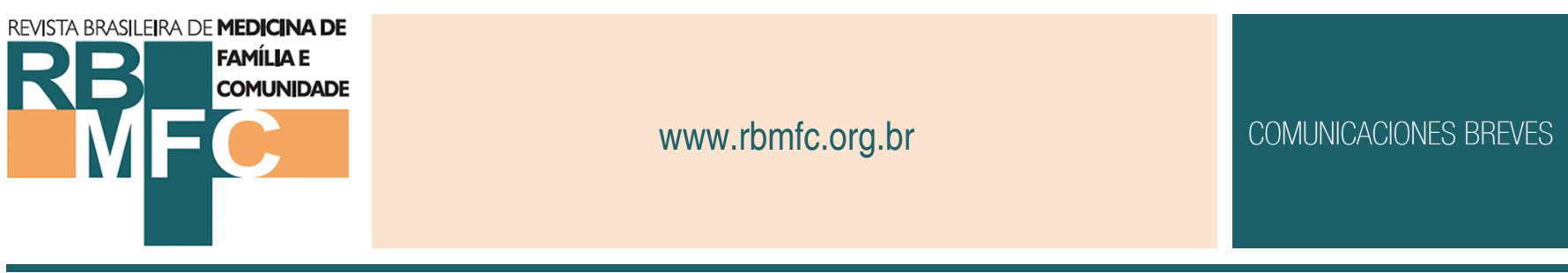

\title{
Declaración de Cali
}

Las Ministras y Ministros participantes en la VII Cumbre Iberoamericana de Medicina Familiar, realizada en Cali, Colombia, con el objetivo de reflexionar sobre el papel de la Medicina Familiar en los Sistemas de Salud basados en Atención Primaria, y específicamente en la atención de situaciones complejas de las personas, las familias y las comunidades como centro de estos Sistemas:

Conscientes de la importancia de la salud y la medicina familiar y comunitaria para la garantía de la cobertura universal en salud en el marco de los Objetivos de Desarrollo Sostenible, y la importancia de hacer un balance regional de la implementación de los compromisos de la declaración de Alma-Ata.

Reconociendo que la declaración de Alma Ata constituye un hito en la historia de las Conferencias internacionales de salud, con el propósito de establecer la Atención Primaria en Salud, como la estrategia fundamental para frenar las inequidades en salud y garantizar derechos en salud a nivel mundial.

Considerando los resultados de la Primera Reunión de la Comisión de Alto Nivel "Salud universal en el siglo XXI: 40 años después de Alma-Alta" con el tema "Lograr la salud universal: desafíos y alianzas estratégicas para la abogacía, el diálogo social y la participación intersectorial".

Reconociendo que la Salud y la Medicina Familiar son esenciales en el cuidado de la salud de las personas durante todo su curso de vida, así como el cuidado de las familias y las comunidades, garantizando servicios de salud con enfoque de derechos, intercultural y de género, y que consideren los factores biológicos, psicológicos, económicos, sociales y culturales.

Considerando lo consignando en las Cartas de San José de Costa Rica (2016), Quito - Ecuador y demás Cartas generadas en las Cumbres anteriores.

Conscientes de los cambios demográficos, la transición epidemiológica, los desastres naturales, el incremento de problemas en salud mental, los cambios en el clima, consumo y hábitos de vida, demográficos y la transición epidemiológica, entre otros fenómenos que determinan la salud de la población de los países; así como de los retos en materia de políticas, planes y programas para la atención integral en salud con enfoque de salud familiar y comunitaria.

Considerando que en septiembre de 2017 se aprobó por los países miembros, en el seno de la Conferencia Sanitaria Panamericana, la Estrategia regional de recursos humanos para el acceso universal a la salud y la cobertura universal de salud (documento CSP29/10) dados los desafíos persistentes, especialmente en lo relativo a la disponibilidad y distribución del personal, la planificación, la gobernanza, la articulación entre los sectores y la formación, de acuerdo con las necesidades del sistema de salud en relación con el acceso universal a la salud y la cobertura universal de salud.

\section{Acordamos:}

1. Promover políticas públicas en salud para el fortalecimiento de los enfoques de salud mental, medicina familiar y comunitaria en el marco de la Atención Primaria en Salud, según las directrices y normas de cada país, así como los programas de formación, capacitación y educación continua.

Rev Bras Med Fam Comunidade. 2018;13(Suppl 1):7-8. http://dx.doi.org/10.5712/rbmfc13(40)1855 
2. Fortalecer la colaboración entre los países participantes, para el intercambio de experiencias y la disponibilidad de recursos humanos con competencias en salud, salud mental y medicina familiar y comunitaria.

3. Aunar esfuerzos entre los países participantes para generar estrategias que respondan de manera oportuna a las necesidades salud de las personas, las familias, las comunidades, la población migrante y en situación vulnerabilidad.

4. Fomentar políticas para la gestión del conocimiento en medicina familiar y comunitaria para incrementar la disponibilidad de evidencia científica de esta especialidad médica, y su impacto económico en los sistemas de salud, fortaleciendo los programas de especialización en medicina familiar.

5. Fortalecer las destrezas y el rol de los médicos familiares en los equipos de salud, para incrementar la resolutividad en el nivel primario de atención, garantizar la atención continua e integral y dinamizar la coordinación intersectorial y la participación comunitaria.

6. Conformar una red de cooperación técnica entre los Ministerios de Salud de los países participantes, con el apoyo de la Organización Panamericana de la Salud, en materia de medicina familiar y atención primaria, que desarrolle al menos los siguientes ejes temáticos: Investigación en el territorio; Impacto económico de la medicina familiar en los sistemas de Salud; salud mental; prevención cuaternaria, atención de desastres, y salud de los migrantes.

7. Solicitar a la Secretaría General Iberoamericana, la inclusión de esta red, en el registro de redes Iberoamericanas, con el fin de involucrar a los demás países de lberoamérica y movilizar recursos técnicos y financieros para el desarrollo de las actividades de la red.

8. Utilizar y aprovechar los resultados de estas acciones de cooperación técnica e intercambio de conocimientos, para el fortalecimiento de las políticas de salud pública y los sistemas de salud de nuestros países.

Firmado en la ciudad de Cali, Colombia, el 14 de marzo de 2018.

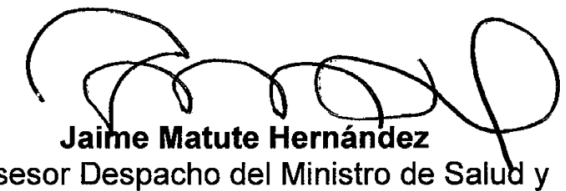

Asesor Despacho del Ministro de Salud y Protección Social de Colombia

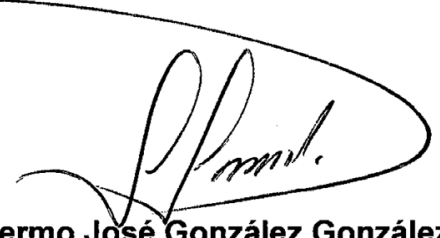

Guillermo José González González

Ministro-Director del Sistema Nacional para la Prevención, Mitigación y Atención de Desastres de Nicaragua

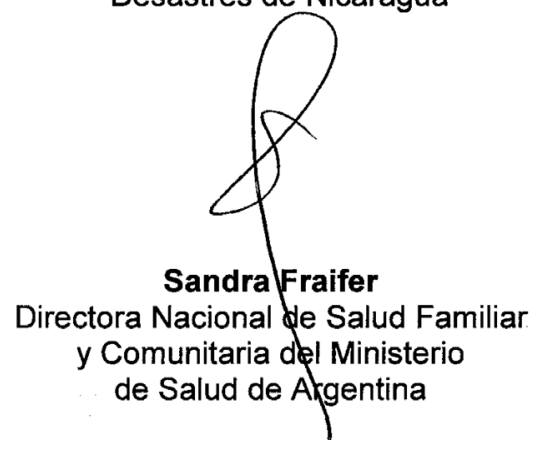

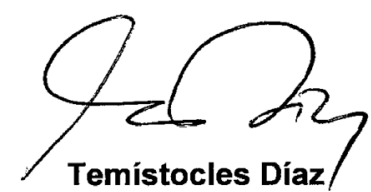

Ministro Consejero de la Presidencia de la República de Panamá

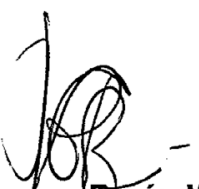

María Teresa Barán Wasilckuk Viceministra de Salud Pública y Bienestar Social de Paraguay

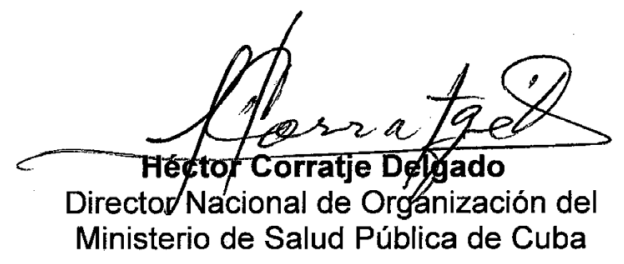

